\title{
Tracking of Triangular References Using Signal Transformation for Control of a Novel AFM Scanner Stage
}

\author{
Ali Bazaei, Member, IEEE, Yuen Kuan Yong, Member, IEEE, S. O. Reza Moheimani, Fellow, IEEE, and \\ Abu Sebastian, Member, IEEE
}

\begin{abstract}
In this paper, we design feedback controllers for lateral and transversal axes of an atomic force microscope (AFM) piezoelectric tube scanner. The controllers are constrained to keep the standard deviation of the measurement noise fed back to the displacement output around $0.13 \mathrm{~nm}$. It is shown that the incorporation of appropriate inner loops provides disturbance rejection capabilities and robustness against dc gain uncertainties, two requirements for satisfactory operation of signal transformation method. Simulations and experiments show significant improvement of steady-state tracking error with signal transformation, while limiting the projected measurement noise.
\end{abstract}

Index Terms-Closed-loop bandwidth, measurement noise, robustification, scanning probe microscopy, sensor fusion, signal transformation, switched control, triangular reference.

\section{INTRODUCTION}

$\mathbf{O}$ BSERVATION, control, and manipulation of matter at very small dimensions have attracted a great amount of attention in nanotechnology [1], [2]. The invention of scanning probe microscopy (SPM) is one of the revolutionary events in nanoscience and nanotechnology [3]-[5]. SPMs promise breakthroughs in areas such as nanometrology [6]-[9], nanolithography [10], [11], material science [12], [13], high-density data storage systems [14]-[16], and nano-fabrication [17]. SPMs are capable of generating 3-D maps of material surfaces on an atomic scale.

Piezoelectric tube scanners are the most commonly used mechanism in commercial AFM to position a sample close to the cantilever probe and to move the sample in a raster pattern during scanning [18], [19]. The tube is fixed at one end and free at the other. Due to the large length-to-diameter ratio of the piezoelectric tube scanner, the free end of the tube experiences

Manuscript received March 18, 2010; revised September 29, 2010; accepted January 05, 2011. Manuscript received in final form February 04, 2011. Date of publication March 24, 2011; date of current version February 01, 2012. Recommended by Associate Editor K. Röpke. This work was supported in part by IBM Zurich Research Labs and by ARC Centre of Excellence for Complex Dynamic Systems and Control.

A. Bazaei, Y. K. Yong, and S. O. R. Moheimani are with the School of Electrical Engineering and Computer Science, The University of Newcastle Australia, Callaghan NSW 2308, Australia (e-mail: ali.bazaei@ newcastle.edu.au, yuenkuan.yong@newcastle.edu.au, reza.moheimani@newcastle.edu.au).

A. Sebastian is with the IBM Zürich Research Laboratory, Rüschlikon CH-8803, Switzerland (e-mail: ase@ zurich.ibm.com).

Color versions of one or more of the figures in this paper are available online at http://ieeexplore.ieee.org.

Digital Object Identifier 10.1109/TCST.2011.2114347 a relatively low mechanical resonance frequency. This makes the tube susceptible to scan-induced vibration. During raster scanning, a triangular waveform is applied to the fast axis ( $x$-axis) and a staircase or ramp signal is applied to the slow axis ( $y$-axis) of the piezoelectric tube scanner. The triangular waveform contains all odd harmonics of the fundamental frequency. The high-frequency content in a fast triangular waveform can excite the tube's resonance and subsequently distort the scanned image.

Accurate tracking of a fast triangular waveform is one of the major challenges not only in SPM [19]-[29] but also in other scanner-based devices such as optical scanners and selective laser sintering (SLS) machines [30], [31]. The performance of the piezoelectric tube scanner is often quantified by its positioning resolution (which is governed by measurement noise), tracking bandwidth and robustness to disturbances [5]. There has been a significant effort to improve the tracking accuracy and speed of piezoelectric tube scanners using feedback control techniques. To track a fast triangular signal, high bandwidth closed-loop controllers have been implemented in many nanopositioning devices [19], [23], [24], [26], [28], [32]-[34]. However, the scanning speed is limited in feedback control systems due to hysteresis, thermal drift, sensor noise, uncertainty, and mechanical vibrations when piezoelectric tubes are used to follow non-smooth triangular trajectories [18]. Capacitive and inductive sensors are commonly used in nanopositioning systems due to their capability of providing simple solution for non-contact, high-resolution measurement. These sensors typically have a noise density of $20 \mathrm{pm} / \sqrt{\mathrm{Hz}}$ [26]. For every hundredfold increment in the closed-loop system bandwidth, the position accuracy of a nanopositioning scanner will decrease tenfold. This potentially degrades the resolution of the scanner, hindering it from performing positioning tasks that require subnanometer resolution. Hence, feedback control methods with limited closed-loop bandwidth are of considerable importance.

Command pre-shaping methods can be considered as a possible way for vibration suppression in an already designed closed-loop control system, leaving the closed-loop bandwidth of the measurement noise unaffected [35]-[44]. However, these methods are not suitable for tracking of time-varying commands such as triangular waveforms or suffer from lack of robustness to plant uncertainties. Iterative learning control (ILC) can also be added as a feed-forward control action in a feedback system to improve the steady-state tracking error for repetitive references without altering the closed-loop bandwidth 


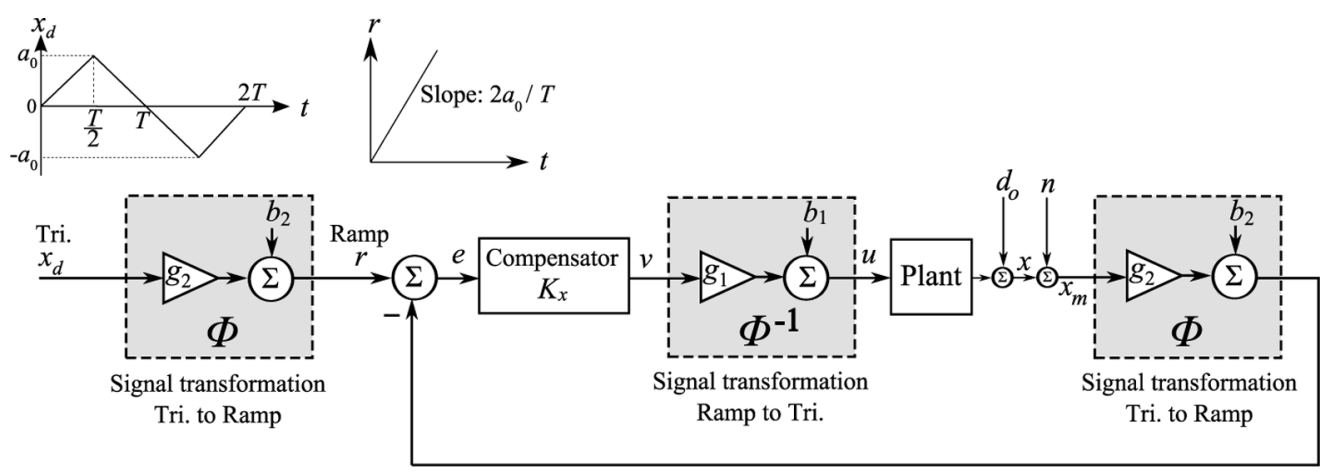

Fig. 1. Schematic diagram of signal transformation method for triangular waveform tracking.

[30], [45]. However, it may require a large number of iterations to converge. Feedback control methods such as repetitive control (RC) for tracking of periodic references introduce large closed-loop bandwidths, which may not be acceptable in the presence of measurement noise. Moreover, the tradeoff between the tracking error and rejection of non-periodic disturbances in $\mathrm{RC}$ systems can cause problems when excessive cross coupling exist between the scanner axes [46], [47].

In [48], the concept of signal transformation was put forward as a novel approach for tracking of triangular waveforms in a nanopositioning system. The method showed significant closed-loop performance improvement compared with an ordinary feedback-control-system having a similar control bandwidth. However, the method is sensitive to DC gain variations and disturbances arising from cross coupling between the two axes.

This paper explains how signal transformation method can be used along with traditional feedback control methods to improve tracking error in an atomic force microscope (AFM) scanner while keeping closed-loop measurement noise below a pre-specified level and providing stability and robustness to dc-gain variations and disturbances.

\section{OBJECTIVES}

An objective of this paper is to evaluate the capability of the signal transformation method in reducing projected measurement noise compared to ordinary feedback systems. ${ }^{1}$ To do this, we maintain the standard deviation of the projected measurement noise around $0.13 \mathrm{~nm}$ at the actual displacement of $x$-axis. The other objective is to provide disturbance rejection capability and robustness when signal transformation is incorporated into the control systems.

\section{SignAL TRANSFORMATION}

Signal transformation approach incorporates appropriate mappings between non-smooth signals (e.g., triangular waveforms) and smooth signals (e.g., ramps) in a control system to

\footnotetext{
${ }^{1}$ To characterize the impact of measurement noise in feedback control systems, the concept of projected noise is introduced. Consider a typical feedback control system designed to control a physical quantity $x$, which is measured by a sensor, that provides a measured signal $x_{m}:=x+n$ for feedback, which is affected by measurement noise $n$. By the projected noise, we mean the direct effect of the measurement noise signal $n$ on the actual controlled output $x$ in the closed-loop feedback system. For linear systems, this effect can be quantified in terms of the noise signal $n$ and the closed-loop transfer function from $n$ to $x$.
}

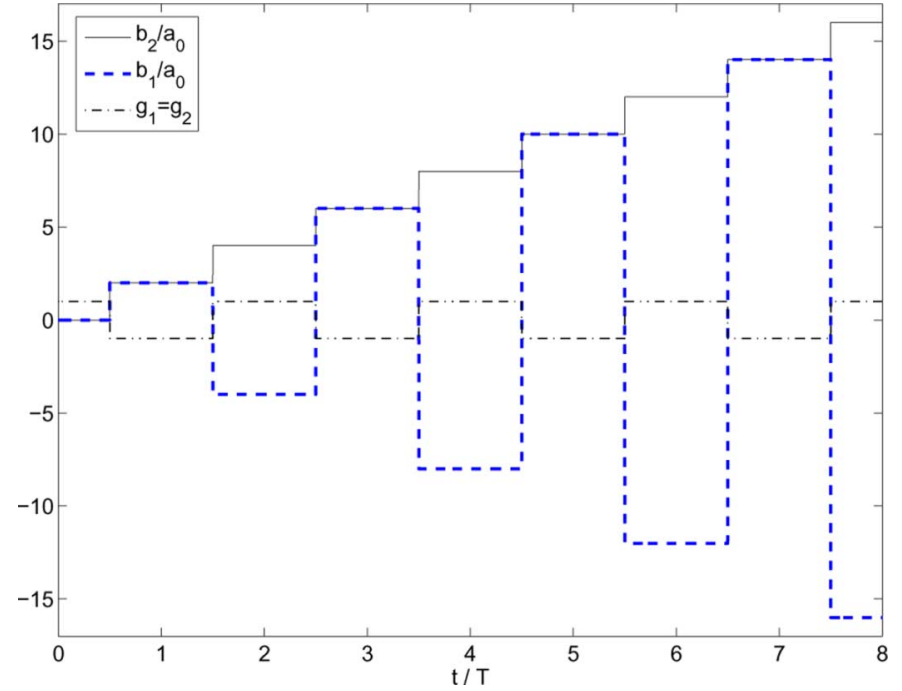

Fig. 2. Gains and bias signals used for triangular waveform tracking.

improve the tracking error while keeping the closed-loop bandwidth low to limit the projected measurement noise [48]. The signal transformation method for control of a single-input-single-output (SISO) plant is described by the hybrid control system shown in Fig. 1, where $\Phi$ and $\Phi^{-1}$ refer to the signal transformation mappings, which in the case of triangular signal tracking use piecewise constant gains $g_{1}$ and $g_{2}$, as well as biases $b_{1}$ and $b_{2}$, as shown in Fig. 2 and can be presented in the following forms:

$$
g_{1}=g_{2}=(-1)^{k}, b_{2}=2 a_{0} k, b_{1}=-(-1)^{k} b_{2}
$$

where $a_{0}$ is the amplitude of the desired triangular waveform $x_{d}$, which has period $2 T$, as shown in the left top insert in Fig. 1, and $k$ is the index of half period defined as

$$
k(t)=\text { floor }\left(\frac{t}{T}+0.5\right) .
$$

The signal transformation blocks, which use $g_{2}$ and $b_{2}$, can convert the non-smooth periodic triangular signal $x_{d}$ into a smooth ramp signal denoted by $r$ in the left top insert in Fig. 1. The signal transformation block between the plant and compensator does the reverse action, i.e., it converts the smooth ramp signal into a non-smooth triangular signal.

To understand how the method works, consider a steady-state ideal situation, where the noise $n$ and output disturbance $d_{o}$ are zero, the plant is a unity gain transfer function, and its output is 
perfectly following the desired triangular signal. Also assume that the compensator contains at least two integrators for perfect tracking of ramp references. In this case, the mapping $\Phi$ acting on the plant output $x$ generates an output signal equal to the ramp reference signal $r$ such that the error signal $e$ vanishes after a transient. The double integral action converts the error signal to a ramp signal $v$ at the compensator's output. The inverse mapping $\Phi^{-1}$ converts the smooth ramp signal $v$ to a non-smooth triangular actuation signal at $u$, which is required to generate a triangular signal at the plant output. Hence, the input/output signals at compensator block are smooth signals with no breaks or discontinuities and the burden of providing appropriate non-smooth trajectories at the actuator, which demands a high control bandwidth in an ordinary feedback system, is done by the signal transformation block. In this way, the compensator can be designed with a smaller closed-loop bandwidth in favor of rejecting the projected measurement noise without deteriorating the steady-state error. The signal transformation method, however, has robustness and disturbance rejection problems, which will be explained in Section IV.

\section{INVESTIGATION OF SySTEM RoBUSTNESS}

In this section, we use simulations to show that the signal transformation method mentioned in Section III can improve the tracking performance of feedback control systems with low closed-loop bandwidth, which translates into low projected noise. We also investigate robustness of the method to DC gain variations and output disturbances using a model obtained for the $x$-axis of an AFM scanner. To do this, we use a model for the $x$-axis of the scanner, obtained after closing a damping loop around a low noise piezoelectric strain-induced voltage sensor to damp the first resonance of the tube (see Section VI-C). The model has zeros at $230 \pm 6000 i,-1180 \pm 876 i$, and -2.1 , and poles at $-1286 \pm 1992 i,-1100 \pm 1497 i$, and -2.3 . A constant gain of $1 / 0.42$ was included at the input to force a unity de gain for the plant in Fig. 1. The compensator in Fig. 1 is a double integrator plus an integrator in the following form:

$$
K_{x}(s)=2.3 \times \frac{50 s+250}{s^{2}}
$$

which provides a gain margin of $23.4 \mathrm{~dB}$, a phase margin of $87^{\circ}$, and reduces the closed-loop bandwidth to $21 \mathrm{~Hz}$. Such a low closed-loop bandwidth can keep the projected measurement noise around $0.13 \mathrm{~nm}$, as shown in Section VI-D. For a $10 \mathrm{~Hz}$ triangular reference with amplitude $a_{0}=2 \mathrm{~V}$ and under different conditions, the resulting closed-loop steady-state errors are shown in Fig. 3. With unity dc gain and no disturbances the signal transformation provides acceptable tracking (compare the thick solid line curve with a $4 \mathrm{~V}$ peak-to-peak triangular reference). However, when the plant dc gain is increased or is reduced twice $(6 \mathrm{~dB})$, which is much less than the gain margin, the error increases unacceptably, as shown in Fig. 3. This shows lack of robustness against variations in the plant dc gain.

The sensitivity of signal transformation method to plant dc gain variations is mainly due to the design of inverse mapping $\Phi^{-1}$, which generates the plant input $u$. To understand this, let the plant's transfer function be a non-unity constant gain. For perfect tracking, the plant input signal, consistent with the reference, should be a triangular signal whose amplitude is different from $a_{0}$. However, the jumps in signal $g_{1}$ have been adjusted to

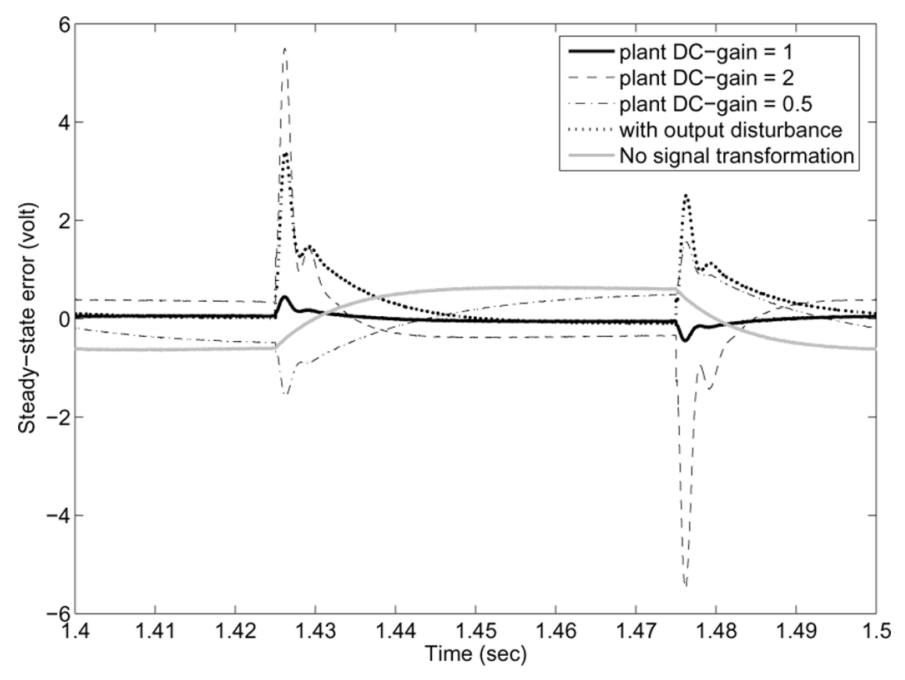

Fig. 3. Demonstration of unacceptable performance of signal transformation method in control of $x$-axis due to plant dc-gain variations, output disturbance, an removal of signal transformation blocks. The steady-state errors are associated with a $10 \mathrm{~Hz}$ triangular reference similar to the top left insert in Fig. 1, whose amplitude is $a_{0}=2 \mathrm{~V}$.

convert a ramp signal to a triangular waveform with amplitude of $a_{0}$.

To evaluate the effect of output disturbances, we set the plant dc gain back to unity and applied a unity amplitude step signal at the exogenous input $d_{o}$ in Fig. 1 while the triangular reference set-point was applied. The resulting steady-state error, plotted in Fig. 3 with the dotted line, shows an undesirable disturbance rejection performance. In this example, the application of signal transformation together with a $21 \mathrm{~Hz}$ control bandwidth cannot achieve acceptable disturbance rejection. Although it is satisfactory for tracking in the absence of disturbances.

To compare the signal transformation method with a 1-degrees-of-freedom (DoF) controller having the same bandwidth, we replaced the signal transformation blocks by unity gains in Fig. 1. The resulting ordinary feedback system with unity plant dc gain and no disturbances has the response labeled "No signal transformation" in Fig. 3, which shows that the $21 \mathrm{~Hz}$ bandwidth without signal transformation is not enough for acceptable tracking of a $10 \mathrm{~Hz}$ triangular reference.

\section{INCORPORATING ROBUSTNESS IN SIGNAL TRANSFORMATION}

In this section, we incorporate an intermediate feedback loop prior to signal transformation blocks, as shown in Fig. 4, to improve the robustness properties mentioned in Section IV. In Fig. 4, the signal transformation mappings denoted by $\Phi$ and $\Phi^{-1}$ are as before, and variables $d_{o x}, n_{x}, v_{c x}, v_{p x}$, and $u_{x}$ stand for output disturbance, measurement noise, capacitive sensor output, piezoelectric strain-induced voltage, and piezoelectric actuation voltage of the $x$-axis, respectively. A low-pass filter $F(s)=(1+s / 1000)^{-1}$ was used to reduce the effect of measurement noise. The intermediate and outer compensators were selected as

$$
\begin{aligned}
K_{i}(s) & =\frac{166.667}{s} \\
K_{i i}(s) & =\frac{50 s+250}{s^{2}} .
\end{aligned}
$$




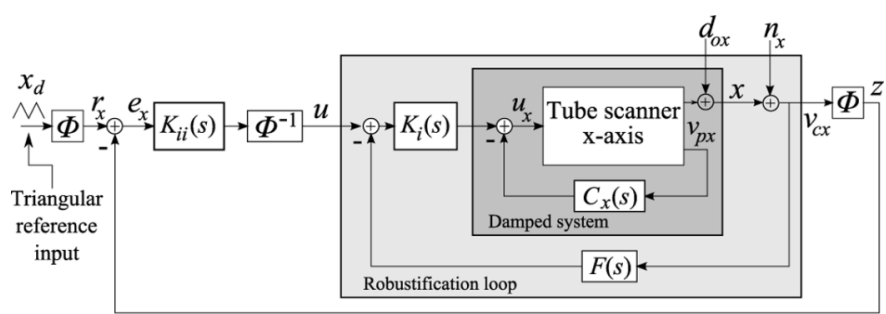

Fig. 4. Schematic diagram of signal transformation method with a robustification loop.
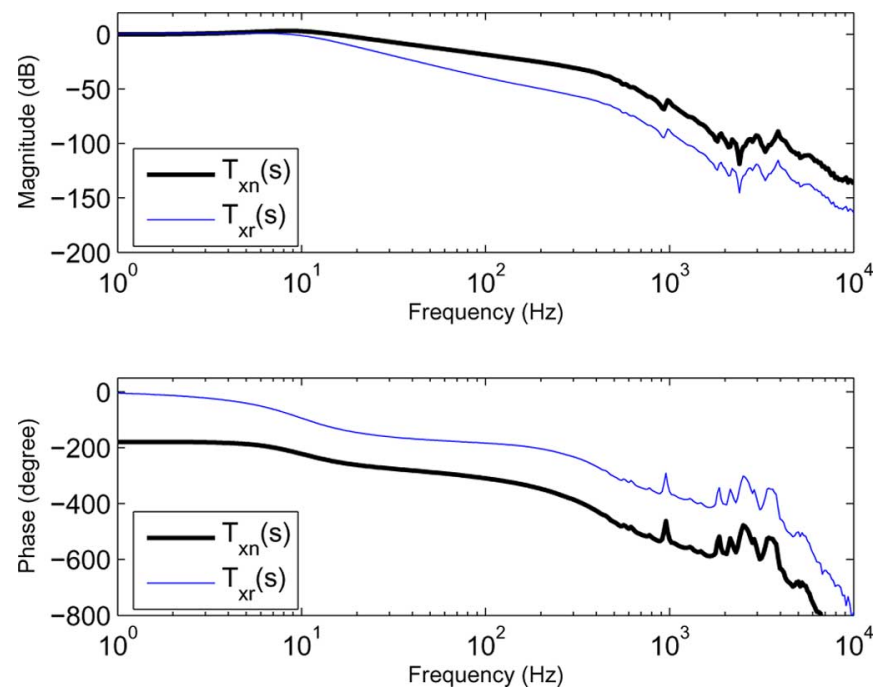

Fig. 5. Frequency responses of closed-loop transfer functions from reference and measurement noise to the real displacement of the $x$-axis.

The compensators $K_{i}(s)$ and $K_{i i}(s)$ were selected such that the overall transfer function from sensor noise $n_{x}$ to the real displacement output signal $x$ has a low bandwidth of $21 \mathrm{~Hz}$ similar to Section IV. This transfer function, whose experimental frequency response is shown in Fig. 5, can be described as

$$
T_{x n}(s):=\frac{x(s)}{n_{x}(s)}=-\frac{K_{i}(s) P_{x}(s)\left[F(s)+K_{i i}(s)\right]}{1+K_{i}(s) P_{x}(s)\left[F(s)+K_{i i}(s)\right]}
$$

where $P_{x}(s)$ is the transfer function of the damped system of $x$-axis, which can be described as

$$
P_{x}(s)=\frac{T_{c x}(s)}{1+C_{x}(s) T_{p x}(s)}
$$

where $T_{c x}(s)$ and $T_{p x}(s)$ stand for the transfer functions from $x$ actuation input voltage to the displacement and piezoelectric strain-induced output voltages of $x$ axis, respectively. The robustification loop by itself (excluding the outer loop) provides a unity dc gain form $u$ to $x$ with a gain margin of $27.7 \mathrm{~dB}$, phase margin of $90^{\circ}$, and bandwidth of $13 \mathrm{~Hz}$. The overall system has a gain margin of $42 \mathrm{~dB}$, phase margin of $55^{\circ}$, and bandwidth of $11.6 \mathrm{~Hz}$ for the forward transfer function from the reference to the displacement output, which is also shown in Fig. 5 and described by the following relationship:

$$
T_{x r}(s):=\frac{x(s)}{r_{x}(s)}=\frac{K_{i}(s) P_{x}(s) K_{i i}(s)}{1+K_{i}(s) P_{x}(s)\left[F(s)+K_{i i}(s)\right]} .
$$

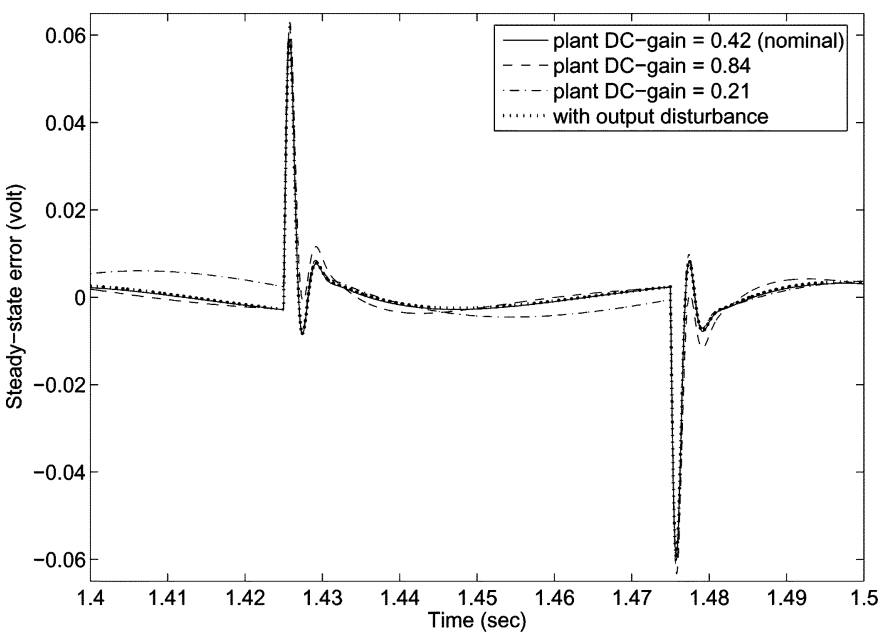

Fig. 6. Effects of plant dc-gain variations and output disturbance on closedloop steady-state response of $x$-axis with robustified signal transformation. The steady-state errors are associated with a $10 \mathrm{~Hz}$ triangular reference similar to the top left insert in Fig. 1, whose amplitude is $a_{0}=2 \mathrm{~V}$.

The simulation results shown in Fig. 6 correspond to closedloop response of the proposed method where the same triangular reference signal and disturbance as in Section IV are used and the plant dc gain in the legends refers to the dc gain of the damped system in Fig. 4. Clearly, the steady-state tracking error remains acceptable in the presence of dc-gain variations of the plant and output disturbance, which shows that the robustification loop can improve the robustness issue of the signal transformation method without deteriorating its benefits (low tracking error with low bandwidth).

\section{EXPERIMENTS}

In this section, signal transformation method with the proposed robustifying scheme is performed on the $x$-axis of the actual scanner for further examination.

\section{A. Description of AFM Scanner}

Fig. 7 shows the NT-MDT NTEGRA scanning probe microscope (SPM) was used to perform experiments reported here. The SPM is configured to operate as an AFM. A protective hood was used as a shield against acoustic noise, electromagnetic fields, and temperature variations. As shown in Fig. 8, the original scanner of the SPM was replaced by a 12-electrode piezoelectric tube scanner [49] which is used for simultaneous sensing and actuation.

External electrode of the piezoelectric tube scanner is segmented into 12 equal sections, as shown in Fig. 9, and the inner electrode is a continuous electrode which is grounded. It has a small continuous electrode at the top of the tube for $z$-axis actuation. One end of the tube is fixed. The free end serves as a stage over which a sample can be placed and its horizontal deflections are measured by two capacitive sensors. Fig. 10 illustrates the wiring of the tube for actuation and sensing in the $x$-axis alone, where $\pm \hat{u}_{x}$ and $\hat{v}_{p x}$ are actuation and piezoelectric strain-induced voltages after and before amplification, respectively. The same wiring is applied to the $y$-axis and is not illustrated for brevity sake. The two outer electrodes on opposite sides are 


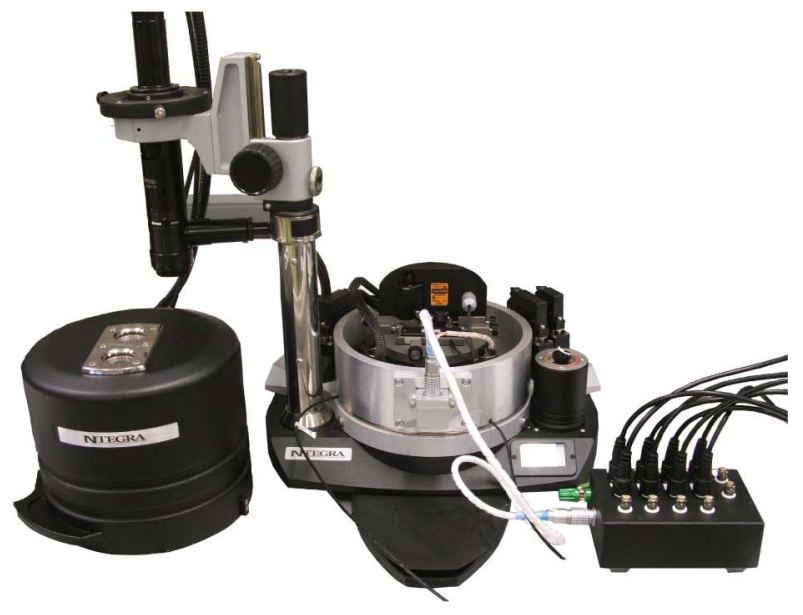

Fig. 7. NT-MDT NTEGRA SPM. The tube scanner is located below the scanning head.

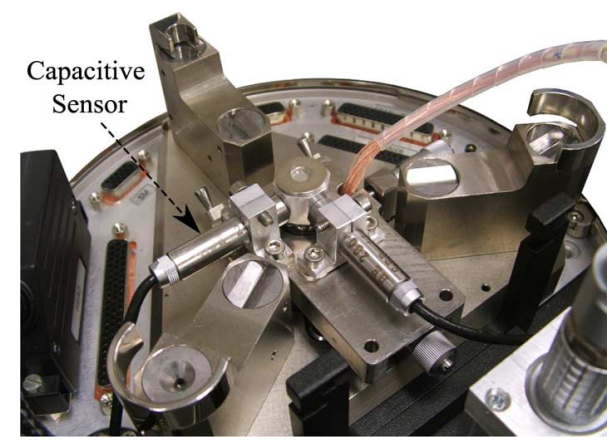

Fig. 8. Tube scanner is installed into the SPM. The two capacitive sensors are mounted at right angles to the target.

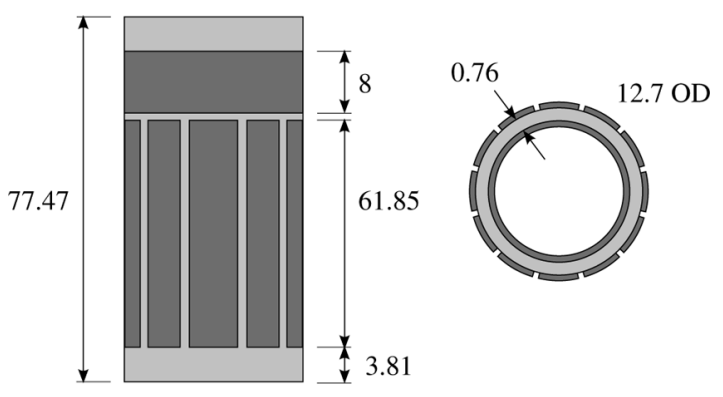

Fig. 9. Segmentation of the electrodes on the piezoelectric tube (dimensions in millimeter).

used for actuation. When voltages with equal magnitudes but opposite polarities $\left( \pm \hat{u}_{x}\right)$ are applied to these electrodes, one side of the tube extends and the opposite side retracts, resulting in bending and top displacement $d_{x}$. The displacement is measured by the voltage signal $v_{c x}$ using a capacitive sensor, whose projected noise is to be kept below a certain level in the feedback systems considered in this paper. The strain experienced on each side of the tube is translated into a voltage at the respective central electrode due to the piezoelectric effect. Due to the symmetry, the voltages induced at the two central electrodes are equal in magnitude but $180^{\circ}$ out of phase. The voltage induced in one electrode is inverted and added to that obtained from the opposite electrode. The resulting signal $\hat{v}_{p x}$, whose

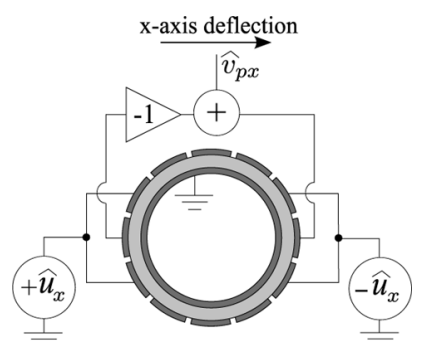

Fig. 10. Simultaneous piezoelectric actuation and sensing for $x$-axis of the tube scanner.

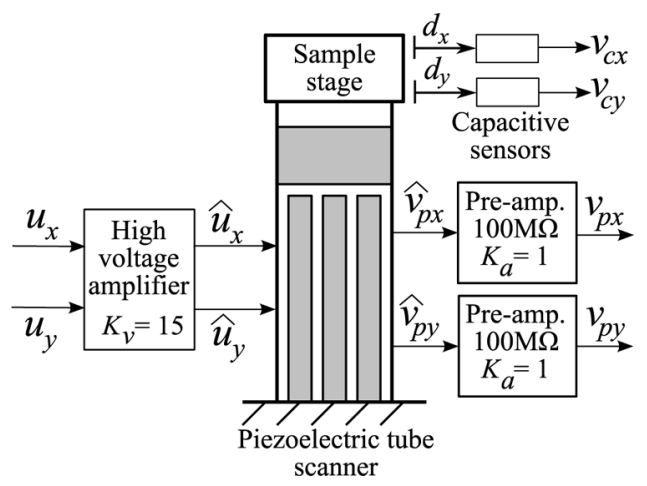

Fig. 11. Block diagram of the experimental setup. $u$ is the input voltage. $\hat{u}$ is the output of the voltage amplifier. $d$ is the displacement of the piezoelectric tube scanner in micrometers. $v_{c}$ is the capacitive sensor output voltage (its sensitivity is $0.32 \mathrm{~V} / \mu \mathrm{m}) . \hat{v}_{p}$ is the strain-induced voltage. $v_{p}$ is the strain-induced voltage after passing through the preamplifier. The FRFs of $\left[T_{p x}, T_{p y}\right]$ plotted in Fig. 12 are from input $\left[u_{x}, u_{y}\right]$ to output $\left[v_{p x}, v_{p y}\right]$. Whereas the FRFs of $\left[T_{c x}, T_{c y}\right]$ are from input $\left[u_{x}, u_{y}\right]$ to output $\left[v_{c x}, v_{c y}\right]$.

noise is negligible, will be used as an auxiliary output in a high bandwidth loop to damp the first resonance of the tube. Actuation and sensing in the $y$-direction can be obtained in a similar manner. For $z$-axis actuation, a voltage is applied to the continuous electrode ( $z$-electrode) near the free end of the tube.

Piezoelectric strain-induced voltages have a first-order high-pass characteristic at low frequencies. This is due to the capacitive nature of the piezoelectric tube and finite input impedance of a measurement device [50]. The transfer function of the strain-induced voltage to the output of a voltage measuring instrument resembles a first-order high-pass filter. To minimize the cutoff frequency of the high-pass filter, piezoelectric strain-induced voltages were fed to low noise preamplifiers (Stanford Research Systems SR560). The input impedance of the preamplifier is $100 \mathrm{M} \Omega$. The measured capacitance of each sensing electrode is $3.2 \mathrm{nF}$. Together with the input impedance of the preamplifier, the cutoff frequency of the high-pass filter is reduced to $0.5 \mathrm{~Hz}$. Although the cutoff frequency is low, accurate positioning at low frequencies using the strain-induced voltage alone could be difficult [19].

Piezoelectric strain-induced voltage has a very low noise profile at high frequencies [19], [26]. The noise density of the strain voltage was reported in [26] to be $16 \mathrm{fm} / \sqrt{ } \mathrm{Hz}$, which is a thousand times smaller than that of a typical capacitive sensor. Therefore, the strain-induced voltage is a preferred choice of sensor for damping resonances of the piezoelectric tube that occurs at high frequencies. Two capacitive sensors, 
(a)
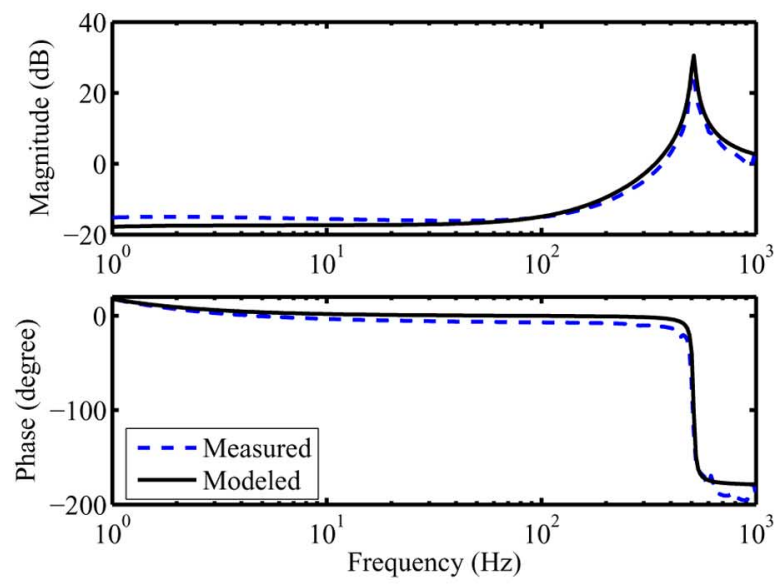

(c)
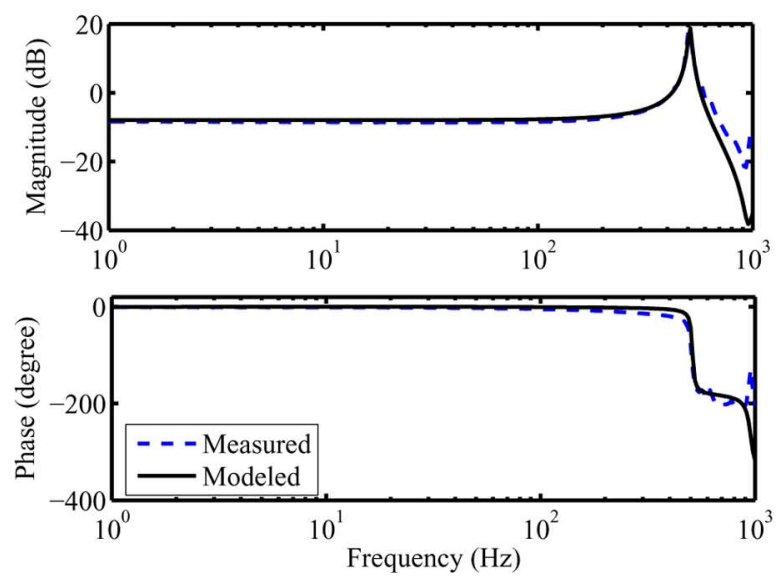

(b)
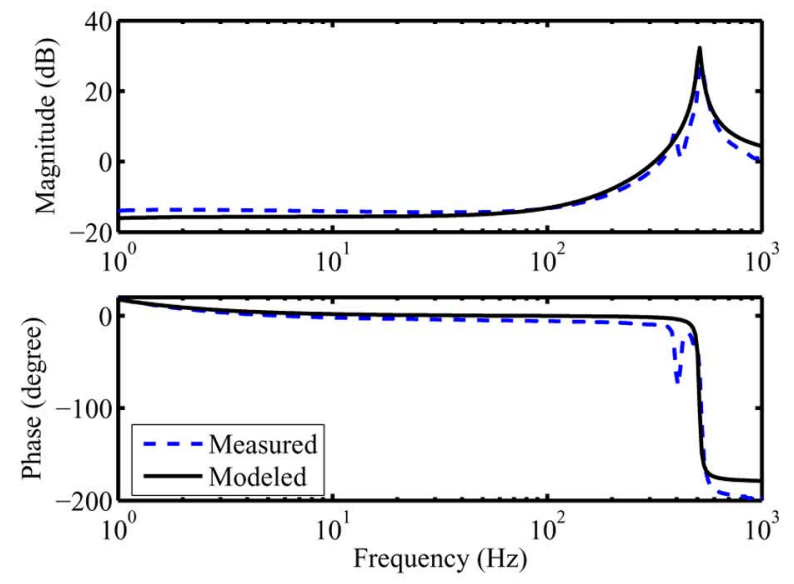

(d)
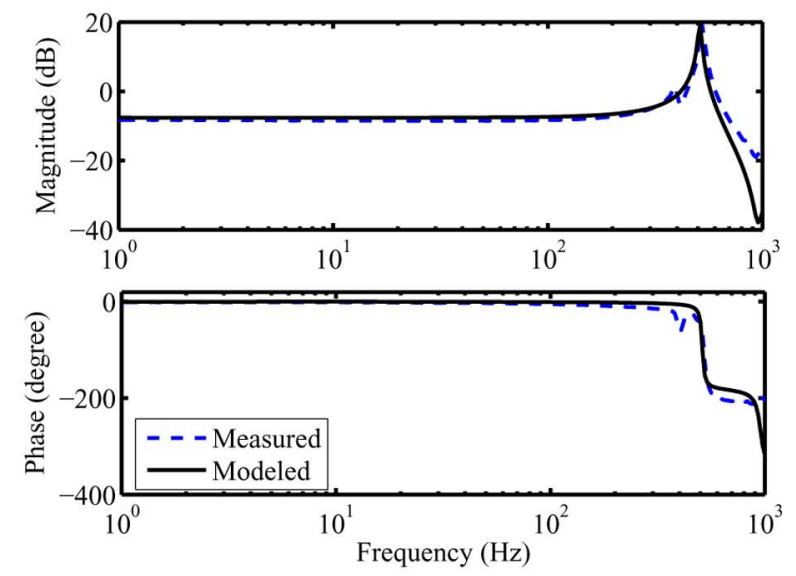

Fig. 12. Measured open-loop FRFs of the piezoelectric tube scanner. (a) and (b) display the FRF of $T_{p x}$ and $T_{p y}$, respectively. (c) and (d) display the FRF of $T_{c x}$ and $T_{c y}$, respectively. A dynamic model is fitted to each of the FRFs. These models capture the first resonant peaks accurately.

which provide displacement measurement, are used for implementation of the horizontal position feedback. As shown in Fig. 8, these capacitive sensors were placed in close proximity to the adjacent surfaces of the sample holder to measure the displacement of the tube scanner along the $x$ - and $y$-axes.

The $x$ - and $y$-axes of the piezoelectric tube were driven by a NANONIS bipolar high voltage amplifier HVA4. The amplifier has a voltage range of $\pm 400 \mathrm{~V}$. A dSPACE-1103 rapid prototyping system was used to implement the $x$ - and $y$-axes feedback controllers in real-time. The $z$-axis displacement was controlled using the AFM's software and circuitry.

\section{B. System Identification and Modeling}

This section presents the procedure undertaken to model the piezoelectric tube scanner used in this work. The model of the device can be identified from the frequency response functions (FRFs) obtained from the apparatus. A bandlimited swept sine input of amplitude $200 \mathrm{mVpk}$, within the frequency range of $1 \mathrm{~Hz}$ to $10 \mathrm{kHz}$, was used to excite the system. The following FRFs were obtained using a HP 35670 A dual channel spectrum analyzer

$$
T_{p x}(i \omega)=\frac{v_{p x}(i \omega)}{u_{x}(i \omega)}, T_{p y}(i \omega)=\frac{v_{p y}(i \omega)}{u_{y}(i \omega)}
$$

and

$$
T_{c x}(i \omega)=\frac{v_{c x}(i \omega)}{u_{x}(i \omega)}, T_{c y}(i \omega)=\frac{v_{c y}(i \omega)}{u_{y}(i \omega)}
$$

where $u_{x}$ and $u_{y}$ are the input voltages applied to the high voltage amplifiers, $v_{p x}$ and $v_{p y}$ are the piezoelectric induced voltages, and $v_{c x}$ and $v_{c y}$ are the capacitive sensor voltages of the $x$ - and $y$-axis, respectively. Fig. 11 shows the block diagram of the experimental setup used for obtaining the FRFs. The measured open-loop FRFs are plotted in Fig. 12. The measured FRFs were approximated by the following third-order LTI models:

$$
\begin{aligned}
& {\left[\begin{array}{l}
T_{p x}(s) \\
T_{p y}(s) \\
T_{c x}(s) \\
T_{c y}(s)
\end{array}\right] \approx \frac{1}{\left(1+\frac{2 \zeta}{\omega_{o}} s+\frac{s^{2}}{\omega_{o}^{2}}\right)\left(1+\frac{s}{2}\right)} \times} \\
& {\left[\begin{array}{c}
0.0675\left(1-\frac{s^{2}}{1200^{2}}\right) s \\
0.0825\left(1-\frac{s^{2}}{1200^{2}}\right) s \\
0.42\left(1-1.276 \times 10^{-5} s+2.774 \times 10^{-8} s^{2}\right)\left(1+\frac{s}{2.1}\right) \\
0.4275\left(1-1.276 \times 10^{-5} s+2.774 \times 10^{-8} s^{2}\right)\left(1+\frac{s}{2.06}\right)
\end{array}\right]}
\end{aligned}
$$


(a)

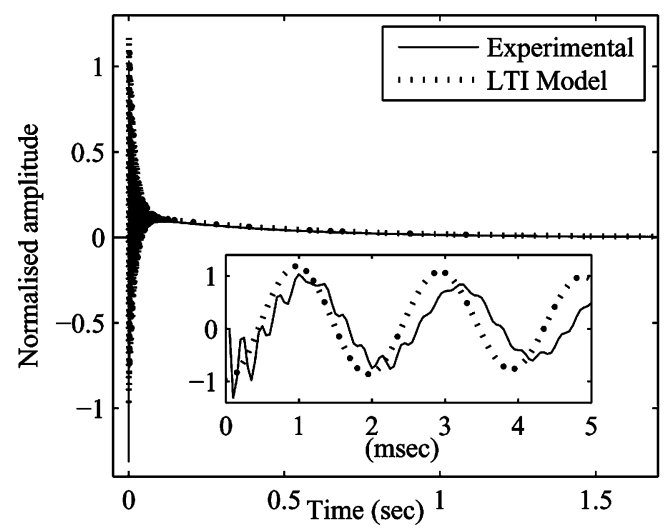

(c)

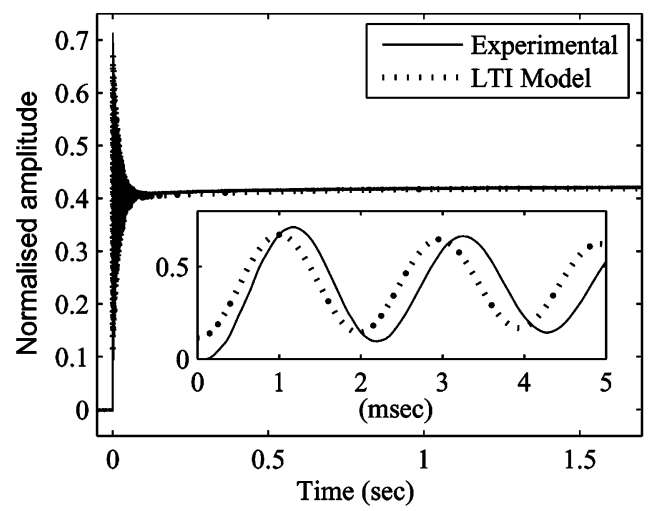

(b)

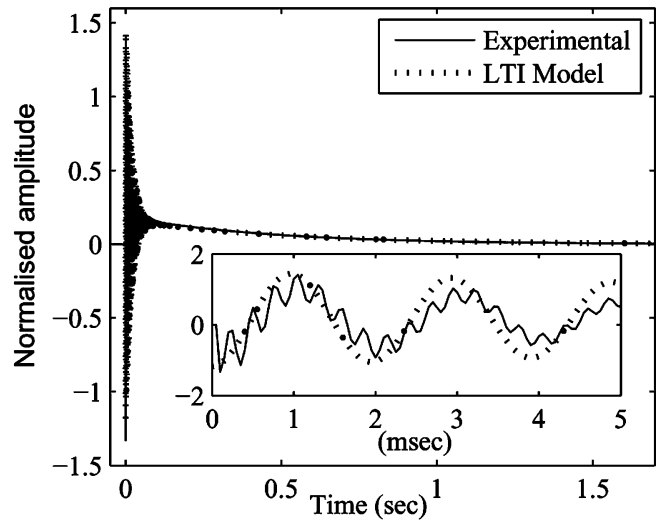

(d)

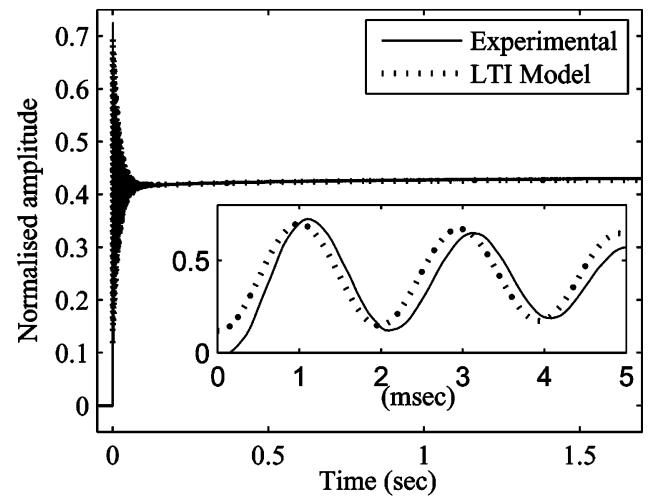

Fig. 13. Experimental step responses of the open-loop system along with those of the LTI model.

where $\omega_{o}=3210.7 \mathrm{rad} / \mathrm{s}$ and $\zeta=0.016$. As shown in Fig. 12, the LTI model roughly approximates the frequency response of the system within the bandwidth of interest. The real pole $s=-2$ was included to cope with the time response of the system to constant inputs in a long run. Fig. 13 shows normalized step responses of the system obtained by individually applying positive step inputs with amplitudes $2 \mathrm{~V}$ at $u_{x}$ and $u_{y}$ and then dividing the measured outputs by $2 \mathrm{~V}$, where the insert in each graph shows a clear view of the response at the initial moments. The step responses of the individual channels of the LTI model, shown in Fig. 13, show that the time response of the LTI model is roughly consistent with the experimental results. The undershoots of the piezoelectric output voltages at the starting moments demonstrates the non-minimum-phase behavior of the system, which justifies the right-half plane zeros in the LTI models for $T_{p x}$ and $T_{p y}$.

\section{Damping Loop}

Our purpose in this section is to damp the frequency response of the scanner without limiting the bandwidth much less than the first resonance frequency. The piezoelectric strain-induced voltages, which have less noise than capacitive sensors, were used as feedback signals to damp the first resonant peak of each axis of the tube scanner. The structure of the damping loop is shown in Fig. 14. To design the controller $C_{x / y}$ for each axis, we use affine parametrization method [51]. Using the transfer functions obtained in Section VI-B for the piezoelectric outputs as the plant and considering all poles of the plant undesirable, affine parametrization method leads to the following poles assignment equation:

$$
\bar{L}(s) A_{o}(s)+\bar{P}(s) B_{o}(s)=\widetilde{E}(s)
$$

where $B_{o}(s)$ and $A_{o}(s)$ are numerator and denominator polynomials of the plant transfer function, respectively, $\widetilde{E}(s)$ is the desired characteristic polynomial after closing the damping loop, and $\bar{P}$ and $\bar{L}$ are the numerator and denominator polynomials of the damping compensator, respectively. Since $A_{0}$ and $B_{o}$ are of order three, selecting degree of two for the compensator polynomials $\bar{P}$ and $\bar{L}$ and degree of five for the desired polynomial $\widetilde{E}$ lead to a unique solution for compensator. The desired closed-loop poles of the damping loops for $x$ - and $y$-axes, which determine $\widetilde{E}(s)$, were selected as

$$
\begin{aligned}
& x \text {-axis: }-1000 \pm 1500 i,-1500 \pm 2000 i,-2.3 \\
& y \text {-axis: }-1000 \pm 4000 i,-1500 \pm 2000 i,-2.15 .
\end{aligned}
$$

To keep an acceptable stability margin, we had to keep one of the desired closed-loop poles around the slow open-loop pole of the plant at $s=-2$. For $x$-axis, which needs to track a fast triangular waveform, the selected desired imaginary poles have more damping in comparison with the $y$-axis, which should track a less steep ramp. Because of unmodeled dynamics, it is desirable for the compensator transfer functions to roll off at high frequencies to avoid spillover effect. Hence, after calculating the compensator transfer functions, we dropped the $s^{2}$ term in the numerator of $C_{x}(s)$ and $s^{2}$ and $s$ terms in the numerator of $C_{y}(s)$ 


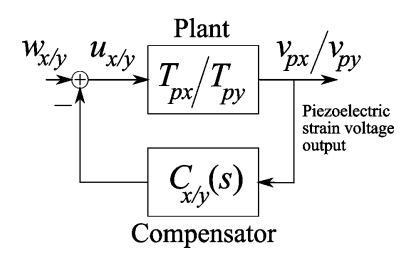

Fig. 14. Feedback architecture of damping loops.

to zero. With these changes, the gain margin of $16.5 \mathrm{~dB}$ and the closed-loop poles changed very little for $x$-axis. For $y$-axis they moved to $-801 \pm 3245 i,-1825 \pm 2227 i$ and -2.15 , which are still around the original ones, however, the gain margin changed to $23 \mathrm{~dB}$ (from $15 \mathrm{~dB}$ ). The resulting $x$ and $y$ damping compensators are as follows:

$$
\begin{aligned}
C_{x}(s) & =\frac{-0.9663(1+0.00114 s)}{1+0.00109 s+4.624 \times 10^{-7} s^{2}} \\
C_{y}(s) & =\frac{-0.4222}{1+0.000533 s+1.035 \times 10^{-7} s^{2}} .
\end{aligned}
$$

The negative signs in the compensators show their consistency with the positive position feedback (PPF) method mentioned in [23]. Using the experimental frequency responses obtained in Section VI-B, the frequency responses of $x$ and $y$ displacement outputs after closing the damping loops along with the openloop responses are shown in Fig. 15. Clearly, the first resonance of each axis has been damped without considerably limiting the bandwidth.

The damped $y$-axis is controlled by an ordinary integral control as shown in Fig. 16, where compensator

$$
K_{y}(s)=\frac{1500}{s}
$$

provides gain margin $8.2 \mathrm{~dB}$ and phase margin $86^{\circ}$. The $y$-axis reference signal $r_{y}$ is a ramp signal whose slope is 512 times less than the slope of the $x$-axis triangular reference. The $x$-axis controllers and the triangular reference signal are as in Section V. The overall noise transfer function $T_{x n}(s)$ for the $x$-axis controller has a bandwidth of $21 \mathrm{~Hz}$ as before. A calibration grating (MikroMasch TGQ1) with a $3 \mu \mathrm{m}$ period, $1.5 \mu \mathrm{m}$ square side and $20 \mathrm{~nm}$ height was used for imaging. A contact mode Cont Al cantilever probe with a resonance frequency of $13 \mathrm{kHz}$ was used to perform the scan. To evaluate the scanning performance of the controllers, a $9.8 \mathrm{~Hz}$ triangular reference signal was applied to the $x$-axis and the aforementioned synchronized ramp signal was applied to the $y$-axis of the piezoelectric tube scanner to generate a $10 \mu \mathrm{m} \times 10 \mu \mathrm{m}$ image (with $256 \times 256$ scan lines). Fig. 17(a) shows the scanned image and the tracking performance of the $x$-axis displacement with signal transformation of the piezoelectric tube scanner. The RMS error of the tracking signal is $80 \mathrm{~nm}$.

\section{Tracking Performance and Noise}

The resolution of the piezoelectric tube is often governed by the sensor noise due to the noise being fed back to the actuator in (a)
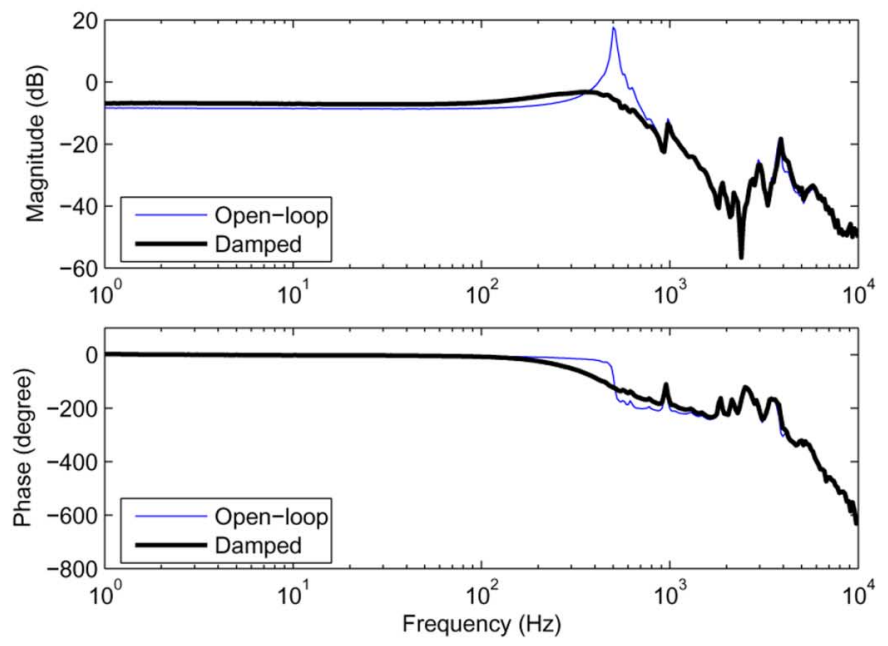

(b)
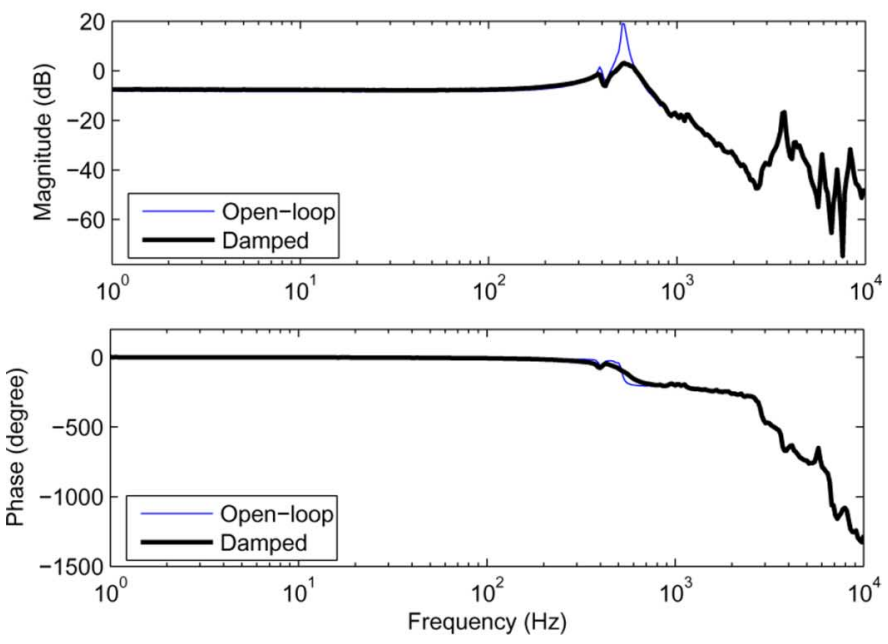

Fig. 15. Frequency responses of displacements before and after the damping loops: (a) $x$-axis and (b) $y$-axis.

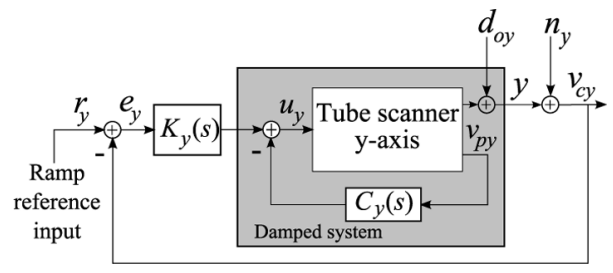

Fig. 16. Schematic diagram for control of $y$-axis.

closed loop systems. This makes open-loop architecture a more attractive solution than that of closed-loop. However, open-loop devices are sensitive to nonlinear effects such as drift and creep. These effects deteriorate the tracking performance and subsequently degrade the image quality generated by the devices.

The signal transformation method presented in this paper ensures that the noise content of the controlled $x$-position signal $x$, in Fig. 4 , is low. To estimate the noise content of the $x$-axis 


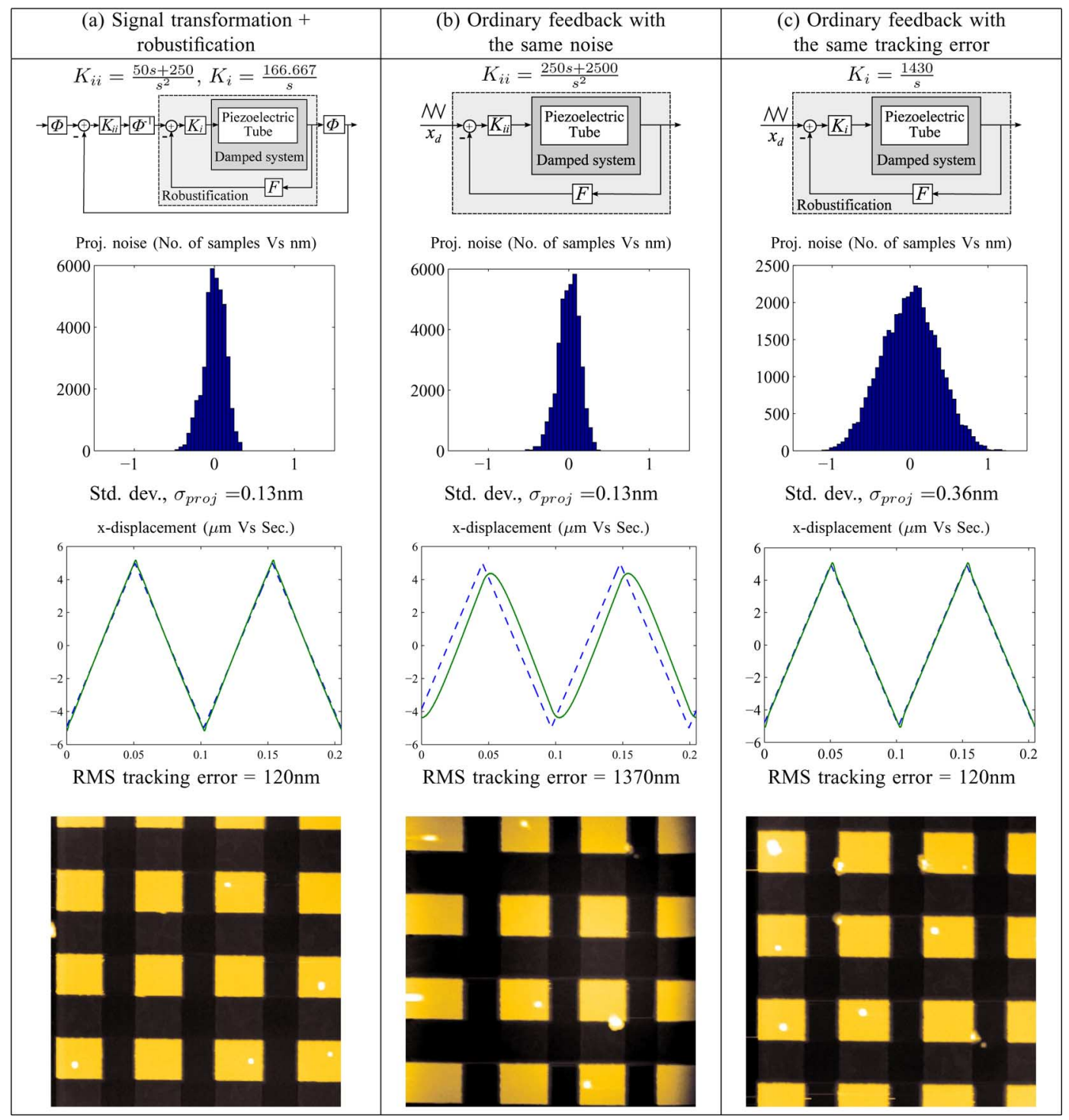

Fig. 17. Comparison of the projected noise, tracking error and AFM image $(10 \mu \mathrm{m} \times 10 \mu \mathrm{m})$ of the three closed-loop systems. (a) Closed-loop system with signal transformation and robustification loop. (b) Closed-loop system without signal transformation. The double integral controller $K_{i i}$ was tuned such that the projected noise is similar to system (a) at the expense of more tracking error. (c) Closed-loop system without signal transformation. The integrator gain was tuned to 1430; therefore the tracking performance is similar to system (a) at the expense of more projected noise. $\sigma_{\text {proj }}$ is the standard deviation of the projected noise. Tracking performance in the $x$-axis of each closed-loop system is illustrated. Triangular reference signal $(--)$ and output displacement $(-)$ are plotted.

displacement, the capacitive sensor output was first recorded as the noise signal $n_{x}$ while the piezoelectric tube remained stationary. Response of noise transfer function $T_{x n}(s)$ to the recorded noise signal was then obtained by simulation as a measure of noise projected into the actual $x$-position and has the histogram shown in Fig. 17(a). The standard deviations of the sensor noise $n_{x}$ and the projected noise are 3.85 and $0.13 \mathrm{~nm}$, respectively. Fig. 18 shows how the inverse mapping $\Phi^{-1}$ converts the smooth signal at the output of the double integrator compensator to a suitable non-smooth actuation signal for the robustified plant during the experiment.

To evaluate efficacy of signal transformation, we now consider an ordinary feedback system with the same level of projected noise for comparison purposes. To do this, we use the control structure shown in Fig. 17(b), which uses a double integrator compensator as $K_{i i}=\left(250 s+2500 / s^{2}\right)$, which was adjusted to provide the same standard deviation of $0.13 \mathrm{~nm}$ for the projected noise. With a the triangular reference signal applied at $x_{d}$ in Fig. 17(b), the resulting steady-state tracking performance of the $x$-axis is as shown in Fig. 17(b), where the root-mean-square (RMS) tracking error is considerably more than that of the signal transformation method. This tracking error is mostly contributed by the low closed-loop bandwidth of the system.

Alternatively, if in the latter system, which does not include signal transformation blocks, we increase the integrator gain of the $x$-channel to 1430 to keep the RMS value of the resulting steady-state tracking error equal to that of the system that includes signal transformation blocks, the standard deviation of the projected noise will increase to $0.36 \mathrm{~nm}$, as shown in Fig. 17(c), which is almost three times more than that obtained with signal transformation. Thus, signal transformation with the proposed robustification loop provides better tracking performance, while keeping 
(a)

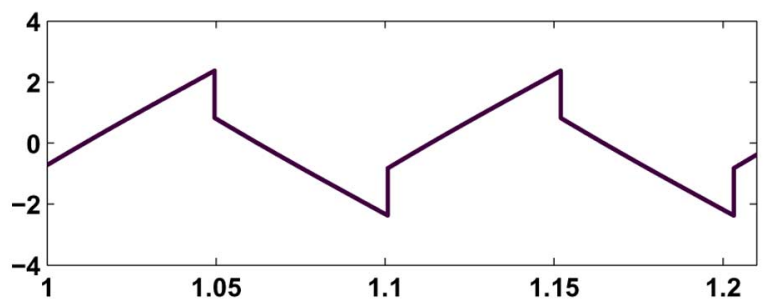

(b)

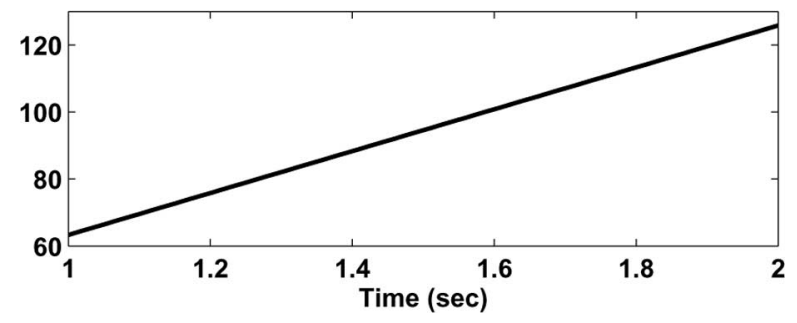

Fig. 18. Operation of signal conversion block $\Phi^{-1}$ in signal transformation method. (a) Output of $\Phi^{-1}$ or input to the robustified plant. (b) Input to $\Phi^{-1}$ or output of the double integrator compensator.

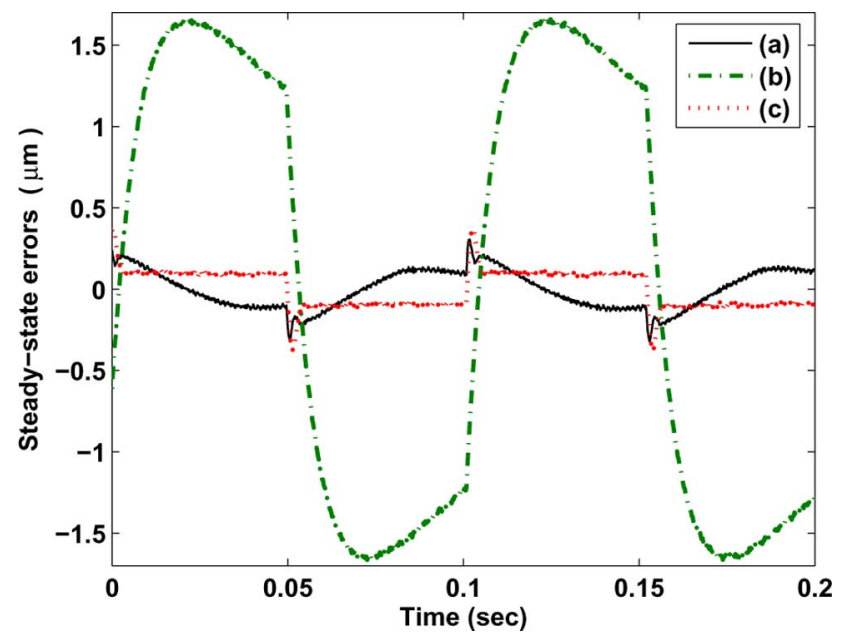

Fig. 19. Steady-state tracking errors associated with the three controllers in Fig. 17.

low projected measurement noise and robustness against disturbance and parameter variations.

For all the three controllers designed above, the corresponding images of the calibration grating and the tracking errors have been included in Figs. 17 and 19, respectively. The image with severe distortions is caused by the poor tracking performance of the second controller. The difference between the image of the first and third controllers is indistinguishable. This is due to the similar tracking performance of the two controllers. The image quality of the two closed loop systems is the same, which is determined by the sensor as well as environmental noise.

\section{CONCLUSION}

Signal transformation method can improve the steady-state error in tracking of a periodic triangular desired signal while limiting the closed-loop control bandwidth to minimize the impact of measurement noise on positioning accuracy. Robustness issue of signal transformation method against dc gain variations and output disturbance was demonstrated and a method was offered to solve this problem. Effectiveness of the proposed method was examined by simulations and experiments on a piezoelectric tube AFM scanner.

\section{ACKNOWLEDGMENT}

The authors would like to thank E. Eleftheriou and H. Pozidis for their support.

\section{REFERENCES}

[1] S. Devasia, E. Eleftheriou, and S. O. R. Moheimani, "A survey of control issues in nanopositioning," IEEE Trans. Control Syst. Technol., vol. 15, no. 5, pp. 802-823, Sep. 2007.

[2] Y. Sugimoto, P. Pou, O. Custance, P. Jelinek, M. Abe, R. Prez, and S. Morita, "Complex patterning by vertical interchange atom manipulation using atomic force microscopy," Science, vol. 322, pp. 413-417, Oct. 2008

[3] B. Bhushan, Nanotribology and Nanomechanics, An Introduction. New York: Springer-Verlag, 2005.

[4] S. Kalinin and A. Gruverman, Scanning Probe Microscopy: Electrical and Electromechanical Phenomena at the Nanoscale. New York: Springer, 2006

[5] S. Salapaka and M. Salapaka, "Scanning probe microscopy," IEEE Control Syst. Mag., vol. 28, no. 2, pp. 65-83, Apr. 2008.

[6] S. Gonda, T. Kurosawa, and Y. Tanimura, "Mechanical performances of a symmetrical, monolithic three-dimensional fine-motion stage for nanometrology," Meas. Sci. Technol., vol. 10, pp. 986-993, 1999.

[7] J.-A. Kim, J. W. Kim, B. C. Park, and T. B. Eom, "Measurement of microscope calibration standards in nanometrology using a metrological atomic force microscope," Meas. Sci. Technol., vol. 17, no. 7, pp. 1792-1800, 2006.

[8] J. Kwon, Y.-S. Kim, K. Yoon, S.-M. Lee, and S. i. Park, "Advanced nanoscale metrology of pole-tip recession with AFM," in Proc. 6th Int. Conf. Scanning Probe Microscopy, Sensors Nanostructures (Ultramicroscopy), 2005, vol. 105, pp. 51-56.

[9] F. Meli and R. Thalmann, "Long-range afm profiler used for accurate pitch measurements," Meas. Sci. Technol., vol. 9, no. 7, pp. 1087-1092, 1998.

[10] K.-B. Choi and J. J. Lee, "Passive compliant wafer stage for singlestep nano-imprint lithography," Rev. Scient. Instruments, vol. 76, pp. 075106-075106, 2005.

[11] D. L. White and O. R. Wood, "Novel alignment system for imprint lithography," Rev. Scient. Instruments, vol. 18, no. 6, pp. 3552-3556, 2000.

[12] K. Miyahara, N. Nagashima, T. Ohmura, and S. Matsuoka, "Evaluation of mechanical properties in nanometer scale using AFM-based nanoindentation tester," in Proc. 4th Int. Conf. Nanostructured Materials (NANO'98)., 1999, vol. 12, pp. 1049-1052.

[13] I. Schmitz, M. Schreinera, G. Friedbachera, and M. Grasserbauer, "Phase imaging as an extension to tapping mode AFM for the identification of material properties on humidity-sensitive surfaces," Appl. Surface Sci., vol. 115, no. 2, pp. 190-198, 1997.

[14] A. Pantazi, A. Sebastian, G. Cherubini, M. Lantz, H. Pozidis, H. Rothuizen, and E. Eleftheriou, "Control of MEMS-based scanning-probe data-storage devices," IEEE Trans. Control Syst. Technol., vol. 15, no. 5, pp. 824-841, Sep. 2007.

[15] A. Pantazi, A. Sebastian, T. A. Antonakopoulos, P. Bächtold, A. R. Bonaccio, J. Bonan, G. Cherubini, M. Despont, R. A. DiPietro, U. Drechsler, U. Dürig, B. Gotsmann, W. Häberle, C. Hagleitner, J. L. Hedrick, D. Jubin, A. Knoll, M. A. Lantz, J. Pentarakis, H. Pozidis, R. C. Pratt, H. Rothuizen, R. Stutz, M. varsamou, D. Wiesmann, and E. Eleftheriou, "Probe-based ultrahigh-density storage technology," IBM J. Res. Dev., vol. 52, no. 4, pp. 493-511, 2008.

[16] A. Sebastian, A. Pantazi, S. O. R. Moheimani, H. Pozidis, and E. Eleftheriou, "Achieving sub-nanometer precision in a MEMS storage device during self-servo write process," IEEE Trans. Nanotechnol., vol. 7, no. 5, pp. 586-595, Sep. 2008.

[17] E. B. Brousseau, F. Krohs, S. Dimov, C. Griffiths, S. Scholz, A. Rees, and S. Fatikow, "Investigation of a new process chain based on atomic force microscopy scratching," in Proc. 4M/ICOMM-The Global Conf. Micro Manuf., 2009, pp. 267-270. 
[18] S. O. R. Moheimani, "Invited review article: Accurate and fast nanopositioning with piezoelectric tube scanners: Emerging trends and future challenges," Rev. Scient. Instruments, vol. 79, Jul. 2008, Article No: 071101 .

[19] I. A. Mahmood, S. O. R. Moheimani, and K. Liu, "Tracking control of a nanopositioner using complementary sensors," IEEE Trans. Nanotechnol., vol. 8, no. 1, pp. 55-65, Jan. 2009.

[20] S. Lining, R. Changhai, R. Weibin, C. Liguo, and K. Minxiu, "Tracking control of piezoelectric actuator based on a new mathematical model," J. Micromechan. Microeng., vol. 14, no. 11, pp. 1439-1444, 2004.

[21] Y.-C. Huang and D.-Y. Lin, "Ultra-fine tracking control on piezoelectric actuated motion stage using piezoelectric hysteretic model," Asian J. Control, vol. 6, no. 2, pp. 208-216, 2004.

[22] A. J. Fleming and S. O. R. Moheimani, "A grounded load charge amplifier for reducing hysteresis in piezoelectric tube scanners," Rev. Scient. Instruments, vol. 76, no. 7, pp. 073707-1-073707-5, 2005.

[23] B. Bhikkaji, M. Ratnam, A. J. Fleming, and S. O. R. Moheimani, "High-performance control of piezoelectric tube scanners," IEEE Trans. Control Syst. Technol., vol. 5, no. 5, pp. 853-866, Sep. 2007.

[24] B. Bhikkaji, M. Ratnam, and S. O. R. Moheimani, "PVPF control of piezoelectric tube scanners," Sensors Actuators: A. Phys., vol. 135, pp. 700-712, Apr. 2007.

[25] S. Aphale, A. J. Fleming, and S. O. R. Moheimani, "High speed nanoscale positioning using a piezoelectric tube actuator with active shunt control," Micro Nano Lett., vol. 2, pp. 9-12, Jan. 2007.

[26] A. J. Fleming, A. Wills, and S. O. R. Moheimani, "Sensor fusion for improved control of piezoelectric tube scanners," IEEE Trans. Control Syst. Technol., vol. 16, no. 6, pp. 1265-1276, Nov. 2008.

[27] A. Fleming and K. Leang, "Evaluation of charge drives for scanning probe microscope positioning stages," in Proc. Amer. Control Conf., Jun. 2008, pp. 2028-2033.

[28] S. Aphale, S. Devasia, and S. O. R. Moheimani, "High-bandwidth control of a piezoelectric nanopositioning stage in the presence of plant uncertainties," Nanotechnol., vol. 19, no. 12, pp. 125503-1-125503-9, Mar. 2008.

[29] A. Payam, M. Yazdanpanah, and M. Fathipour, "Design of a feedforward controller for AFM nanopositioning based on neural network control theory," presented at the IEEE Int. Conf. Nano/Micro Eng. Molecular Syst., Shenzhen, China, 2009.

[30] J.-Y. Yen, Y.-C. Yeh, Y.-H. Peng, and J.-F. Lee, "Application of the continuous no-reset switching iterative learning control on a novel optical scanning system," Mechatronics, vol. 19, no. 1, pp. 65-75, 2009.

[31] D. Zhiqiang, Z. Zude, A. Wu, and C. Youping, "A linear drive system for the dynamic focus module of SLS machines," Int. J. Adv. Manuf. Technol., vol. 32, no. 11-12, pp. 1211-1217, 2007.

[32] G. Schitter, P. Menold, H. Knapp, F. Allgöwer, and A. Stemmer, "High performance feedback for fast scanning atomic force microscopes," Rev. Scient. Instruments, vol. 72, no. 8, pp. 3320-3327, 2001.

[33] S. Salapaka, A. Sebastian, J. P. Cleveland, and M. V. Salapaka, "High bandwidth nano-positioner: A robust control approach," Rev. Scient. Instruments, vol. 73, no. 9, pp. 3232-3241, 2002.

[34] Y. K. Yong, S. Aphale, and S. O. R. Moheimani, "Design, identification and control of a flexure-based XY stage for fast nanoscale positioning," IEEE Trans. Nanotechnol., vol. 8, no. 1, pp. 46-54, Jan. 2009.

[35] O. J. M. Smith, Feedback Control Systems. New York: McGraw-Hill, 1958.

[36] N. C. Singer and W. P. Seering, "Preshaping command inputs to reduce system vibration," J. Dyn. Syst., Meas., Control, vol. 112, no. 1, pp. 76-82, 1990.

[37] J. Hyde and W. Seering, "Using input command pre-shaping to suppress multiple mode vibration," in Proc. IEEE Int. Conf. Robot. Autom., 1991, vol. 3, pp. 2604-2609.

[38] G. Mimmi and P. Pennacchi, "Pre-shaping motion input for a rotating flexible link," Int. J. Solids Structures, vol. 38, no. 10-13, pp. 2009-2023, 2001.

[39] E. Gilberta and I. Kolmanovskyb, "Nonlinear tracking control in the presence of state and control constraints: A generalized reference governor," Automatica, vol. 38, no. 12, pp. 2063-2073, 2002.

[40] P. Pennacchi, "Robustness of command input preshaping technique applied to residual vibration reduction," Shock Vibr., vol. 11, no. 3/4, pp. 377-382, 2004.

[41] T. Sugie and H. Suzuki, "Robust reference shaping of periodic trajectories for systems with state/input constraints using impulse and step responses," in Proc. IEEE Conf. Dec. Control, Dec. 2004, vol. 5, pp. $5511-5516$.
[42] S. Rhim, S. G. Lee, and T. G. Lim, "Model-based reference trajectory generation for tip-based learning controller," J. Mechan. Sci. Technol., vol. 19, no. 1, pp. 357-363, 2005.

[43] H. Suzuki and T. Sugie, "Off-line reference shaping of periodic trajectories for constrained systems with uncertainties," IEEE Trans. Autom. Control, vol. 53, no. 6, pp. 1531-1535, Jul. 2008.

[44] K. Kogiso and K. Hirata, "Reference governor for constrained systems with time-varying references," Robot. Autonomous Syst., vol. 57, no. 3 , pp. 289-295, 2009.

[45] S. Tien, Q. Zou, and S. Devasia, "Iterative control of dynamics-coupling-caused errors in piezoscanners during high-speed afm operation," IEEE Trans. Control Syst. Technol., vol. 13, no. 6, pp. 921-931, Nov. 2005.

[46] G. Pipeleers, B. Demeulenaere, F. Al-Bender, J. De Schutter, and J. Swevers, "Optimal performance tradeoffs in repetitive control: Experimental validation on an active air bearing setup," IEEE Trans. Control Syst. Technol., vol. 17, no. 4, pp. 970-979, Jul. 2009.

[47] U. Aridogan, Y. Shan, and K. K. Leang, "Design and analysis of discrete-time repetitive control for scanning probe microscopes," J. Dyn. Syst., Meas., Control, vol. 131, no. 6, pp. 061103-1-061103-12, 2009.

[48] A. Sebastian and S. O. R. Moheimani, "Signal transformation approach to fast nanopositioning," Rev. Scient. Instruments, vol. 80, pp. 076101-076101, Jul. 2009.

[49] S. O. R. Moheimani and Y. K. Yong, "Simultaneous sensing and actuation with a piezoelectric tube scanner," Rev. Scient. Instruments, vol. 79, no. 7, pp. 073702-073702, Jul. 2008.

[50] S. O. R. Moheimani and A. J. Fleming, Piezoelectric Transducers for Vibration Control and Damping. Germany: Springer, 2006.

[51] G. Goodwin, S. Graebe, and M. Salgado, Control System Design. Englewood Cliffs, NJ: Prentice-Hall, 2001.

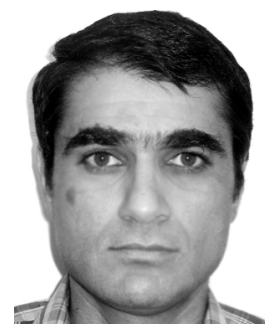

Ali Bazaei (M'11) received the B.Sc. and M.Sc. degrees from Shiraz University, Shiraz, Iran, and the $\mathrm{Ph} . \mathrm{D}$. degrees from University of Western Ontario, London, ON, Canada, and Tarbiat Modares University, Tehran, Iran, in 1992, 1995, 2004, and 2009, respectively, all in electrical engineering.

From September 1995 to January 2000, he was an Instructor with Yazd University, Yazd, Iran. From September 2004 to December 2005, he was a Research Assistant with the Department of Electrical and Computer Engineering, University of Western Ontario, London, ON, Canada. He is currently a Research Academic with the School of Electrical Engineering and Computer Science, The University of Newcastle, Australia. His research interests include the general area of nonlinear systems including control and modeling of structurally flexible systems, friction modeling and compensation, and neural networks.

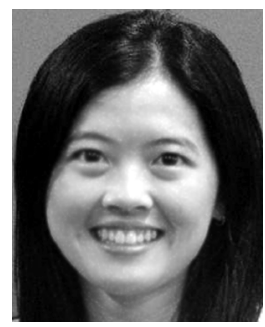

Yuen Kuan Yong (M'09) received the Bachelor of Engineering degree (1st Class Honors) in mechatronic engineering and the Ph.D. degree in mechanical engineering from the University of Adelaide, Adelaide, Australia, in 2001 and 2007, respectively.

She is currently a Research Academic with the Centre for Complex Dynamic Systems and Control (CDSC), The University of Newcastle, Callaghan, Australia. Her research interests include the design and control of nanopositioning systems, finite element analysis (FEA) of smart materials and structures, sensing and actuation, and high-speed atomic force microscopy.

Dr. Yong was a recipient of the 2008 IEEE/ASME International Conference on Advanced Intelligent Mechatronics (AIM) Best Conference Paper Finalist Award. She is a member of the Technical Program Committee of AIM and the International Conference on Manipulation, Manufacturing, and Measurement on the Nanoscale (3M-NANO). 


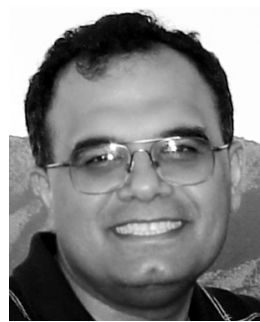

S. O. Reza Moheimani (M'92-SM'00-F'11) received the Ph.D. degree in electrical engineering from University of New South Wales at the Australian Defence Force Academy, Canberra, Australia, in 1996.

Since 1997, he has been with University of Newcastle, Callaghan, Australia, where he is currently a Professor and Australian Research Council Future Fellow with the School of Electrical Engineering and Computer Science. He has served on the editorial boards of a number of journals, including IEEE TRansactions on Control Systems TeChNology, IEEE/ASME TRANSACTIONS ON MECHATRONICS AND CONTROL ENGINEERING PRACTICE.

Prof. Moheimani was a recipient of the 2007 IEEE TRANSACTIONS ON Control Systems Technology Outstanding Paper Award and the 2009 IEEE Control Systems Technology Award. He is a Fellow of the Institute of Physics. His current research interests include applications of control and estimation in nanoscale positioning systems for scanning probe microscopy, control of microactuators in MEMS, and data storage systems.

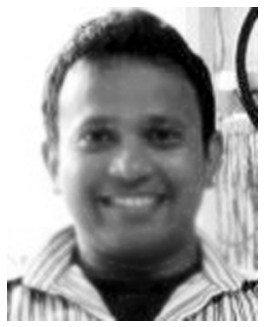

Abu Sebastian (M'03) received the B.E. (Hons.) degree in electrical and electronics engineering from Birla Institute of Technology and Science, Pilani, India, in 1998, and the M.S. and Ph.D. degrees in electrical engineering from Iowa State University, Ames, in 1999 and 2004, respectively.

He is currently a Research Staff Member WITH IBM Research-Zurich, Switzerland, where he has been involved in the research on dynamics and control at the nanometer scale. His research interests include microcantilever-based devices and enabling technologies, such as nanometer-scale sensing and nanopositioning, as well as novel memory concepts, such as probe-based storage and phase change memory.

Dr. Sebastian was a corecipient of the 2009 IEEE Control Systems Technology Award and the 2009 IEEE Transactions on Control Systems Technology Outstanding Paper Award. He is on the editorial board of the Journal Mechatronics. 